\title{
Event-driven Spectrum-Aware Clustering in Cognitive Radio Sensor Networks
}

\author{
Mustafa Ozger Ozgur B. Akan \\ Next-generation and Wireless Communication Laboratory (NWCL) \\ Department of Electrical \& Electronics Engineering \\ Koc University, Istanbul, Turkey \\ Email: \{mozger, akan\}@ku.edu.tr
}

\begin{abstract}
Wireless sensor networks (WSN) with dynamic spectrum access (DSA) capability, namely cognitive radio sensor networks (CRSN), is a promising solution for spectrum scarcity problem. Despite improvement in spectrum utilization by DSA capability, energy-efficient solutions for CRSN are required due to resource-constrained nature of CRSN inherited from WSN. Clustering is an efficient way to decrease energy consumption. Existing clustering approaches for WSN are not applicable in CRSN and existing solutions for cognitive radio networks are not suitable for sensor networks. In this paper, we propose an event-driven clustering protocol which forms temporal cluster for each event in CRSN. Upon detection of an event, we determine eligible nodes for clustering according to local position of nodes between event and sink. Cluster-heads are selected among eligible nodes according to node degree, available channels and distance to the sink in their neighborhood. They select one-hop members for maximizing the number of two-hop neighbors that are accessible by one-hop neighbors through cluster channels to increase connectivity between clusters. Clusters are between event and sink and are no longer available after the end of the event. This avoids energy consumption due to unnecessary cluster formation and maintenance overheads. Performance evaluation reveals that our solution is energy-efficient with a delay due to spontaneous cluster formation.
\end{abstract}

\section{INTRODUCTION}

Inefficient spectrum utilization of traditional fixed frequency assignment approach has triggered the use of dynamic spectrum access (DSA) schemes in wireless communications. Communication technology enabling DSA is cognitive radio (CR) [1]. Cognitive radios have the capability to sense spectrum bands in order to detect empty spectrum portions. With this capability, CRs can utilize spectrum vacancies opportunistically by changing their operating parameters [2]. Hence, the opportunistic spectrum access (OSA) capability overcomes the spectrum scarcity challenge and increases spectrum utilization efficiency.

Wireless sensor nodes equipped with cognitive radio have revealed a new network paradigm which is called cognitive radio sensor networks (CRSN) [3]. Sensor nodes can benefit from the advantages of DSA so that they can communicate intermittently over the licensed bands which is owned by primary users. CRSN nodes detect available channels by spectrum sensing and determine communication channel by spectrum decision and change their operation frequencies by spectrum hand-off if primary users appear on the communication channel. By these functionalities, cognitive radios collaborate with neighbors in order to deliver event samples from event region to the sink in multi-hop manner in such a dynamic radio environment.

In addition to addressing spectrum management challenges by CR capabilities, CRSN imposes energy and hardware limitation challenges inherited from WSN. The solutions for WSN do not consider CR functionalities and hence do not address $\mathrm{CR}$ challenges. On the other hand, the existing solutions for cognitive radio network do not take into account energy and hardware challenges. Even though there are extensive studies on WSN and CRN, CRSN has been receiving interest from the community lately. The recent existing works focus on channel management scheme [4], packet size optimization [5], and reliability and congestion control [6].

Clustering is one of the most important research issues of CRSN that is not investigated thoroughly. Clustering is a structured way to manage topology effectively and to increase the system capacity and stability [7]. There are several studies on clustering for wireless sensor networks and wireless ad-hoc networks [8]-[13]. In these approaches, clustering divides adhoc network into self organized structures each managed by cluster-heads. Those act as a central entity in their corresponding cluster for the efficient and reliable data transmission. Clusters handle the communication in an organized manner by arranging inter-cluster and intra-cluster communication. In WSNs, cluster-heads are selected according to the node ID [14], residual energy [15] and weight of nodes [16]. These clustering studies do not address the dynamic spectrum access challenges, hence, they are not applicable in CRSN regime.

Clustering for cognitive radio networks requires additional constraint which is the condition of grouping nodes according to similar vacant bands in spatial neighborhood. This situation in clustering is termed as spectrum-aware clustering. Clustering in opportunistic spectrum access system requires taking into account local variations of the licensed user activities [18]-[23]. Formed clusters are valid if the nodes forming the cluster have common channels. However, dynamic radio environment requires frequent re-clustering due to the variations of spectrum availabilities.

In sensor networks, packets are generated by the event detecting nodes. These packets create traffic from event to sink. Furthermore, they are transmitted to the sink over available spectrum bands due to CR capability. Hence, sensor 
nodes need coordination in order to reliably deliver these packets in dynamic radio environment. Spectrum-aware clustering provides spectrum sharing by grouping nodes according to their available channels. Furthermore, exploiting eventdriven nature of sensor networks, we achieve energy efficiency by utilizing sources when communication exists. Hence, in this paper we propose event-driven spectrum-aware clustering (ESAC) protocol for CRSN. The contributions of this protocol are itemized as follows:

- ESAC forms clusters after event detection and they are maintained until the end of the event. This brings energy efficiency due to avoiding re-clustering by event-driven clustering approach. This property is consistent with event-driven nature of CRSN.

- Our protocol forms clusters not in the entire network but between event and sink. Therefore, we avoid unnecessary cluster formation which increases energy consumption in resource-constrained CRSN.

- ESAC forms an event routing corridor between event and sink by constructing spectrum-aware clusters by maximizing the number of available channels, one-hop and two-hop member nodes that have common channels for a cluster. By considering to maximize accessible two hop neighbors, we form connected clusters.

The remainder of this paper is organized as follows: Section II presents the related work and Section III explains the network model and assumptions. In Section IV, we describe our protocol ESAC by explaining determination of eligible nodes, clustering algorithm and control overheads of ESAC. We present simulation results in Section V and conclude the paper in Section VI.

\section{RELATED WORK}

Research community has been paying attention to the clustering issue in cognitive radio networks. One of the most important works [18] addresses spectrum heterogeneity and scalability challenge by proposing a distributed coordination scheme. In this scheme, secondary users construct groups according to locally common available channels. This approach offers a distributed group setup and maintenance algorithms. In [19], the network is partitioned into clusters by neighbor nodes sharing local common channels. Clusters are connected to form the network. Furthermore, it proposes a hybrid MAC protocol where channel access time is divided into superframes which have intervals for inter-cluster and intra-cluster communication as well as neighbor discovery. [20] uses graph theory for spectrum opportunity clustering (SOC) and assigns control channel to each cluster so that each node in cluster can communicate within the cluster through local common channels. The recent work [21] presents network topology and spectrum availability as bipartite graph. Every node constructs bipartite graphs with its one-hop neighbors and available channels. Biclique graph is obtained from these bipartite ones according to metrics such as maximum edge, maximum node and maximum edge one sided cardinality. These approaches do not use common channel over the entire network, control channel is assigned to the each cluster in the network by the clustering technique. Through the assigned control channel intra-cluster communication is performed. [22] constructs minimal number of clusters in cognitive radio networks using affinity propagation (AP) message-passing technique. ROSSDGA and ROSS-DFA are distributed clustering approaches offered in [23]. They form robust clusters by providing interand intra-cluster connectivity.

The recent work by [24] suggests a spectrum-aware clustering scheme to enable efficient communication in CRSN by intra-cluster aggregation and inter-cluster relaying and also finds optimal node number in a cluster.

Despite vast amount of clustering approaches in WSN, the idea of clustering between event and sink is first proposed in [17], namely Event-to-Sink Directed Clustering (ESDC). It suggests forming clusters in corridor between the event and the sink in WSN. However, this clustering scheme is not applicable to CRSN since WSN nodes are not aware of CR functionalities and it does not address spectrum management challenges.

Despite the significant amount of clustering approaches on cognitive radio networks, they do not address the challenge of limited energy resource and hardware capacity of sensor nodes. Thus, energy-efficient clustering solution is required to address the limited energy resource challenge as well as opportunistic spectrum access challenges.

\section{Network Model And Assumptions}

Primary and secondary users coexist in a typical CRSN. Primary users (PUs) can utilize their licensed bands directly, however, cognitive radios can operate on these bands in an opportunistic manner [3]. Spectrum readings of neighbor sensor nodes are correlated since transmission range of primary users are comparable to cognitive radios [21].

CR nodes are equipped with single half-duplex transceiver so that transmission and listening are not done simultaneously. There are $C$ non-overlapping orthogonal channels. Each channel has a unique ID and the vacant spectrum bands are detected by spectrum sensing. We assume that channel sensing mechanism returns perfect results and vacant spectrum bands do not change during clustering.

Two nodes can exchange control information through common control channel. It is available for secondary users (SU) at any time [25]. Secondary users access this channel using CSMA/CA protocol. Overlay spectrum is used as interference avoidance model, i.e., SUs use portions of the spectrum not used by the primary users (PU) [2]. Furthermore, nodes know their physical locations by utilizing localization algorithm.

Neighbor discovery is provided by the common control channel signaling periodically. By this signaling, all nodes know one-hop and two-hop neighbors and their vacant channels. In this paper, we are interested in CRSN in which nodes are stationary. Hence, one-hop and two-hop neighbors do not change unless they deplete resources. Let $N_{i}^{1}$ denote onehop neighbor list of node $i$ and $N_{i}^{2}$ two-hop neighbor of it. $C_{i}$ represents the vacant channels of the node $i$. Table I 
TABLE I: Notations For Clustering Protocol

\begin{tabular}{c|l}
\hline \hline Notation & Explanation \\
\hline$N$ & Number of nodes in the network \\
\hline$S$ & Set of nodes in the network \\
\hline$N_{i}^{k}$ & $k$ hop neighbor list of node $i$ \\
\hline$C_{i}$ & Available channel list of node $i$ \\
\hline$d_{i, j}$ & Euclidean distance between nodes $i$ and $j$ \\
\hline$d_{i}^{e}$ & Eligible node degree of node $i$ \\
\hline$P_{i}$ & Weight for cluster-head selection , $\left|C_{i}\right| \mathrm{x} d_{i}^{e}+10 / d_{i, s i n k}$ \\
\hline$C N_{i}[k]$ & $\begin{array}{l}\text { Cluster nodes whose cluster-head is node } i \text { at cluster forma- } \\
\text { tion iteration } \mathrm{k}\end{array}$ \\
\hline$C C_{i}[k]$ & $\begin{array}{l}\text { Common channels for the cluster constructed by the cluster- } \\
\text { head } i \text { at cluster formation iteration } \mathrm{k}\end{array}$ \\
\hline$C D_{i}[k]$ & $\begin{array}{l}\text { Max. eligible two-hop neighbors accessible through a channel } \\
\in C C_{i}[k] \text { and } C N_{i}[k] \text { at cluster formation iteration } \mathrm{k}\end{array}$ \\
\hline$\left.N^{\prime} i\right)$ & Cluster member nodes whose cluster-head $i$ \\
\hline$C(i)$ & Cluster channels whose cluster-head $i$ \\
\hline$R$ & Event radius in meters \\
\hline$D_{e s}$ & Distance between event and sink in meters \\
\hline$r$ & Cognitive radio transmission range in meters \\
\hline$T_{c l s}$ & $\begin{array}{l}\text { Time in msec to form cluster by eligible nodes after detection } \\
\text { of an event }\end{array}$ \\
\hline$E_{c}$ & $\begin{array}{l}\text { Total energy consumed in mJ due to control signaling to form } \\
\text { cluster by eligible nodes after detection of an event }\end{array}$ \\
\hline
\end{tabular}

shows the notations and their explanations which are useful for explaining the clustering protocol.

\section{EVENT-DRIVEN SPECTRUM-AWARE CLUSTERING (ESAC)}

In this section, we explain the proposed event-driven spectrum-aware clustering algorithm for CRSN. In ESAC, event detecting nodes become eligible for clustering. Those nodes send eligibility request message to non-eligible one-hop neighbors and this process continues until the request message reaches to sink. In the mean time, eligible nodes form clusters with their eligible one-hop neighbors. Clusters are maintained until the end of the event. Hence, there are two phases of our protocol. First phase is to determine the eligible nodes. The second phase is to form cluster according to the spectrumawareness of the nodes. These are explained individually in the following subsections.

\section{A. Determination of Eligible Nodes For Clustering}

Clusters are formed only when an event takes place, i.e., event triggers our protocol. The first step of determining the eligible nodes is the event detection. The event detecting nodes become eligible for clustering directly. Afterwards, Eligibility For Clustering REQuest (EFC_REQ) messages are sent by these nodes to their one-hop neighbor through common control channel. Nodes that receive this message determine whether they join clustering. The condition for becoming eligible for clustering is to be located closer to the sink and farther to the event than the EFC_REQ sender. The new eligible nodes send EFC_REQ to their non-eligible one-hop neighbors and the process continues until EFC_REQ reaches the sink. Non-eligible nodes could not send EFC_REQ, hence, noneligible nodes confine further expansion of the eligible region. Algorithm 1 outlines the eligibility process for clustering. We determine eligible nodes in a distributed manner by Algorithm 1 .
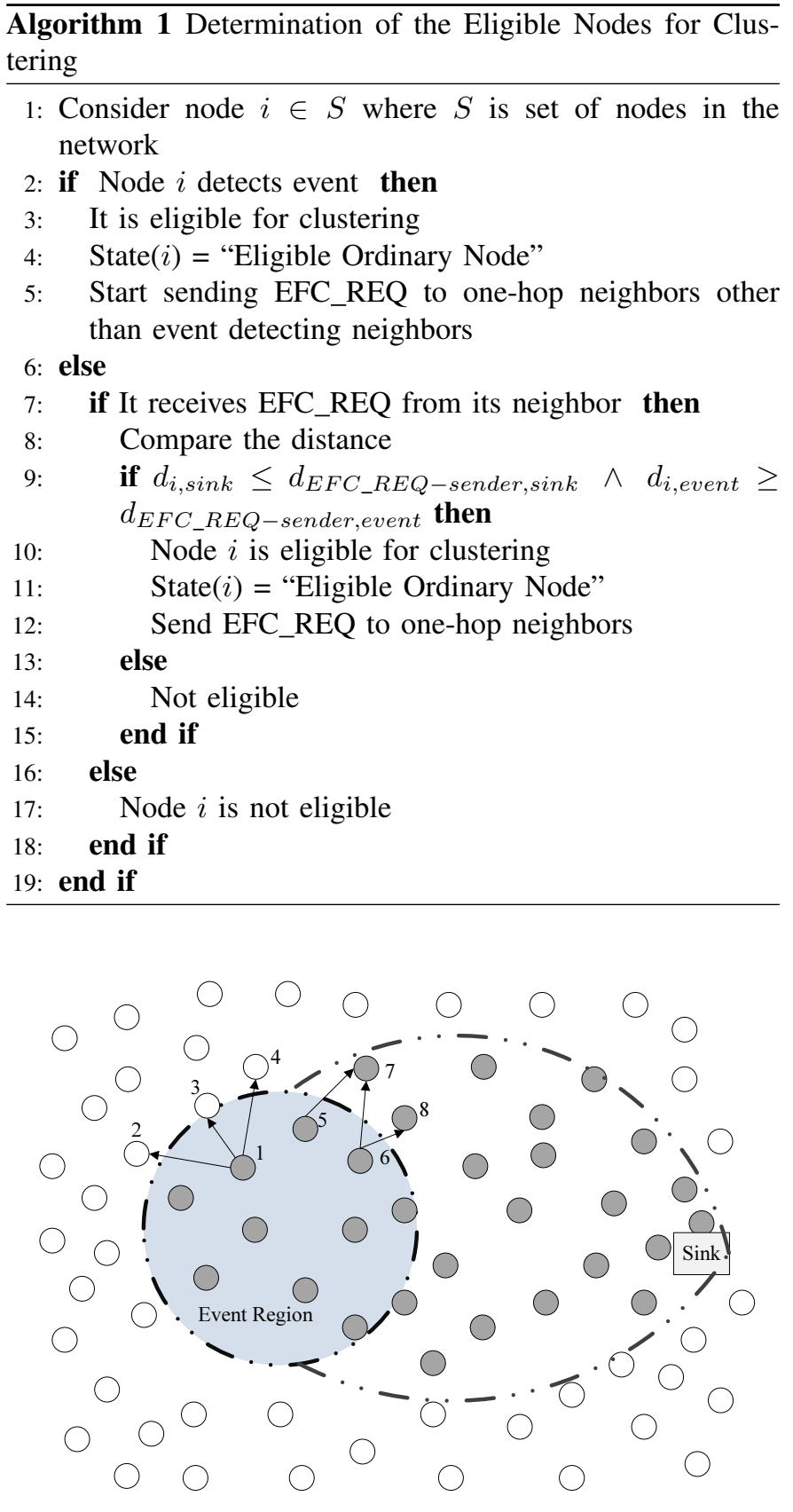

Fig. 1: Illustration of an eligibility scenario

An eligibility scenario is illustrated in Fig. 1. Algorithm 1 is applied and the eligible nodes in the network are shown by filled circles. Eligibility region and the event region are bounded by dashed lines according to Algorithm 1. In this scheme, the event detecting nodes become eligible instantly. The nodes in the event detecting region start sending EFC_REQ immediately to their non-eligible one-hop neighbors and this request disseminates through eligible nodes to the sink. Only eligible nodes send EFC_REQ to their onehop neighbors. For example, node 1 sends EFC_REQ to its 
neighbors 2, 3, and 4 as shown in Fig. 1. However, nodes 2, 3, and 4 cannot join the clustering since they are not closer to the sink than the node 1 . Node 6 sends EFC_REQ to nodes 7 and 8 . The node 7 cannot join clustering by the request coming from node 6 since node 7 is farther to the sink. However, node 7 joins the clustering due to the EFC_REQ from the node 5. State of the eligible nodes is determined to be "Eligible Ordinary Node".

A node knows its neighbors, their locations and available channels. This is periodically updated by neighbor discovery process. If a node receives an EFC_REQ message by one of its neighbors, the sender already knows if the request receiver is eligible. However, the nodes that are eligible or noneligible for clustering inform their neighborhood by Eligibility For Clustering REPly (EFC_REP) message upon receiving an EFC_REQ message.

\section{B. Clustering Algorithm}

Our approach brings a new perspective for clustering in CRSN. The motivation of clustering in sensor network is to create organizational structures. In this work, we try to group the nodes according to their spectrum availabilities while maintaining communication between intra-cluster and inter-cluster nodes which can be degraded by primary users' activities. Only the nodes that are appointed as eligible form clusters. In our approach, clusters are not maintained in the network if event detecting nodes do not further sample the event.

If an eligible node knows the eligibility condition of its neighbors, it initiates clustering process which is explained in Algorithm 2. The clustering algorithm is inspired by [20]. Our clustering protocol is cluster-head first protocol and there is an additional constraint that cluster-heads maximize the number of two-hop members that they can reach by its one-hop members through the cluster channels. By cluster-head first algorithm, we try to decrease control overhead for constructing the clusters and by considering two-hop members, we aim to form non-isolated clusters. Eligibility for clustering and spectrum-aware clustering processes work consecutively such that eligible nodes immediately start forming clusters after having full knowledge about the eligibility of its one-hop members.

In dynamic radio environment, the clusters are formed according to the spectrum availabilities. The nodes which have similar vacant spectrum band are grouped in the same cluster in the same neighborhood. In our clustering scheme, every eligible node is assigned to a weight which is calculated as $P_{i}=\left|C_{i}\right| \times d_{i}^{e}+10 / d_{i, \text { sink }}$. The node $i$ with highest weight $P_{i}$ in its neighborhood is chosen as cluster-head or the node which has the highest weight in any of its neighbor list becomes cluster-head. By this weighting method, we try to select the nodes which have higher eligible node degree and available channel, and which are closer to the sink in its neighborhood.

The cluster-head forms the cluster by selecting appropriate vacant channels and one-hop neighbors subject to maximizing the number of two-hop neighbors that can be reached by that cluster-head by the cluster members through the cluster channel set. If a node $i$ has the highest $P_{i}$ value in its one-hop neighborhood, it tries to maximize the product of three terms for the constructed cluster, which is $\left|C N_{i}\right| \times\left|C C_{i}\right| \times\left|C D_{i}\right|$.

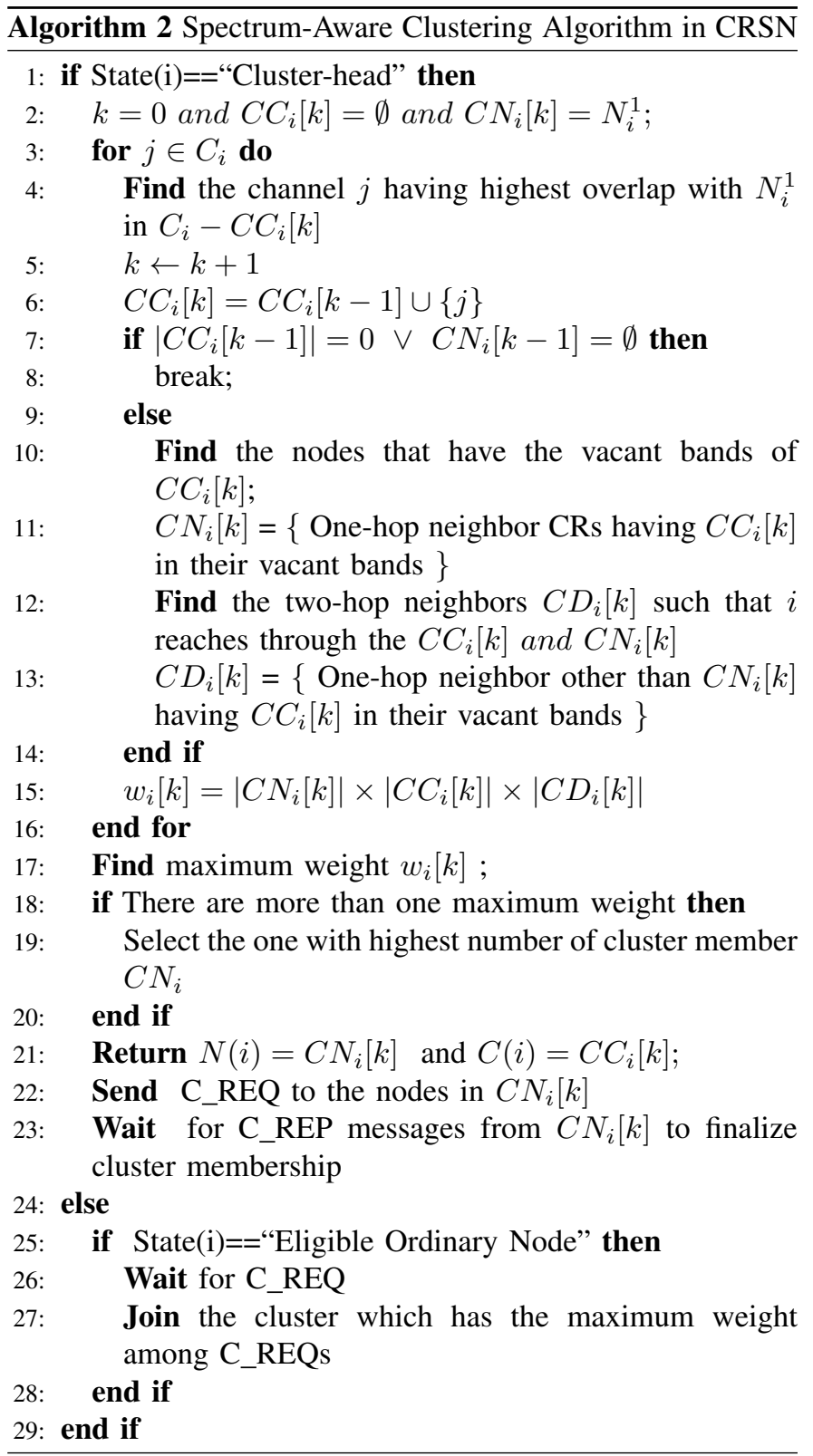

In the clustering procedure, cluster-head node $i$ firstly determines the weight of every channel $C_{i}$ which is the number of one-hop neighbors that node $i$ can reach through the channels in $C_{i}$. Node $i$ firstly adds the channel that has the highest overlap with the one-hop neighbors to $C C_{i}[1]$ where [1] means the first iteration for the algorithm. The weight of each channel is determined by the number of onehop neighbor nodes having this channel in their available channel list. If two or more channels have the same number of weight, we break tie by choosing the lowest numbered one. 
The channel that has the highest weight is added to $C C_{i}[1]$ and the nodes that have this channel in their available channel list are added to $C N_{i}[1]$. We find the maximum number of twohop neighbors that the node $i$ can access through that channel by the nodes in $C N_{i}[1]$, and these nodes are added to $C D_{i}[1]$. The weight of first iteration is $w_{i}[1]$ which is the product of $\left|C N_{i}[1]\right|,\left|C C_{i}[1]\right|$ and $\left|C D_{i}[1]\right|$. In the second iteration, $C C_{i}[2]$ becomes $C C_{i}[1] \cup\{j\}$ where $j$ is the second channel that has the highest overlap with $N_{i}^{1} . C N_{i}[2]$ contains the one-hop neighbor nodes having the vacant bands of $C C_{i}[2]$. $C D_{i}[2]$ is found by determining accessible two-hop neighbors by the nodes in $C N_{i}[2]$ through channels in $C C_{i}[2]$. The weight of this iteration is found as in the previous weight calculation. This process continues until $k^{\text {th }}$ step which results in $C N_{i}[k-1]=\emptyset$ or $\left|C C_{i}[k-1]\right|=0$. In the end, cluster-head $i$ chooses the iteration which has the highest weight. Finally, $N(i)$ is set of cluster nodes constructed by node $i$ and $C(i)$ is the channels common for the nodes in $N(i)$. The algorithm steps can be seen in Algorithm 2.

Fig. 2 shows a tree constructed by cluster-head node 1 in order to determine cluster members and channels. In the first row, two branches denote the vacant spectrum bands of node 1 . Available channels of the node are $A$ and $B$. The second row shows the one-hop eligible nodes having available channels $A$ and $B$. Node 1 has the neighbor nodes 2 and 3 having channel $A$ and the neighbors nodes 2, 3 and 4 having channel $B$. The third row shows the eligible two-hop neighbor nodes. For example, the one-hop neighbor 2 has one-hop neighbor nodes of 5,6 and 7 having available channel of $A$. Since node 1 has two available channels, there are at most two iterations shown in Table II. The first iteration is on the channel $B$ since the number of one-hop neighbors having channel $B$ is greater than that of channel $A$. Afterwards, channel $A$ is added to cluster channel. The nodes having channels $A$ and $B$ are selected as the cluster member for second iteration. Two-hop neighbors having at least one of the iteration channels are the members of $C D_{1}[2]$. According to Algorithm 2, iterations for clusters are calculated and tabulated in Table II. Since second iteration has the highest weight, the cluster member nodes and cluster channels of cluster-head node 1 are $N(1)=\{2,3\}$ and $C(1)=\{B, A\}$.

Firstly, the node 1 with the highest weight in its onehop neighborhood is selected as cluster-head and according to clustering Algorithm 2, it determines the cluster structure and sends Cluster REQuest message (C_REQ) to the nodes in $N(1)$. The nodes receiving this request acknowledge clusterhead that they are now members of cluster constructed by node 1 if cluster weight of node 1 is greater than the other received cluster weight. In other words, if a node receives more than one C_REQs, it accepts the request that has the highest weight. The nodes that are not member of any cluster can become a cluster-head itself. Cluster formation by clusterhead is completed if it knows the condition of eligible one-hop members via Cluster REPly (C_REP) messages.

In this algorithm, we are maximizing the products of number of nodes and channels in the cluster, and the num-

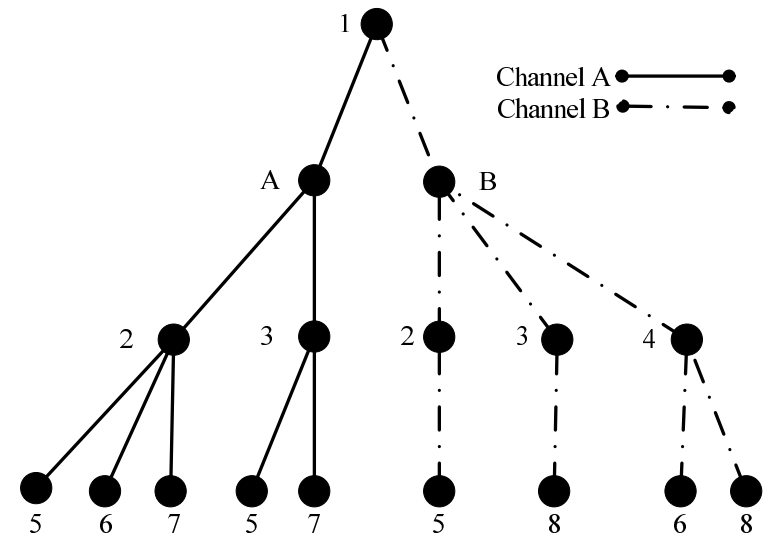

Fig. 2: Tree of a cluster-head

TABLE II: Iterations for Cluster-head node 1 in Fig. 2

\begin{tabular}{|c|c|c|c|c|}
\hline \hline Iteration & $C C_{1}[k]$ & $C N_{1}[k]$ & $C D_{1}[k]$ & $w_{1}[k]$ \\
\hline$k=1$ & $\{B\}$ & $\{2,3,4\}$ & $\{5,6,8\}$ & 9 \\
\hline$k=2$ & $\{B, A\}$ & $\{2,3\}$ & $\{5,6,7,8\}$ & 16 \\
\hline
\end{tabular}

ber of two-hop neighbors that cluster members can directly communicate by cluster channels. By this way, we compromise between common channels, cluster size and inter-cluster connectivity.

\section{Control overheads of ESAC}

In literature, the clustering schemes in cognitive radio maintain the organizational structures in the entire network disregarding event occurrences. Hence, nodes are organized for data transmission at the cost of energy. In our scheme, clusters are formed from scratch upon detection of an event. This requires exchange of control information in order to generate clusters in the corridor between event and sink. These overheads are itemized as follows:

- EFC_REQ is sent by eligible nodes to further determine the eligible nodes closer to the sink starting from event detecting nodes.

- Nodes send EFC_REP to inform one-hop neighbors whether if they are eligible for clustering.

- Cluster-head nodes send C_REQ messages to the cluster members according to Algorithm 2.

- Members validate their membership by sending C_REP message to their cluster-heads.

In order to form clusters, an eligible node except event detecting one sends EFC_REQ, EFC_REP and C_REP or C_REQ according to the node's weight in its neighborhood. Event detecting nodes do not send EFC_REP message since they are directly eligible.

\section{Performance Evaluation}

The performance of our protocol is evaluated in terms of eligible nodes ratio, and delay, total energy consumption, energy efficiency and connectivity. We perform simulations by using MATLAB. 


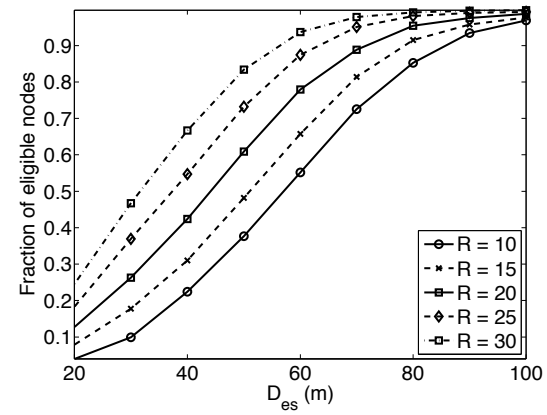

(a)

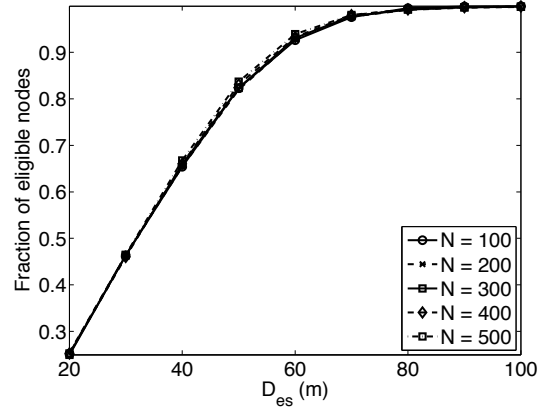

(b)

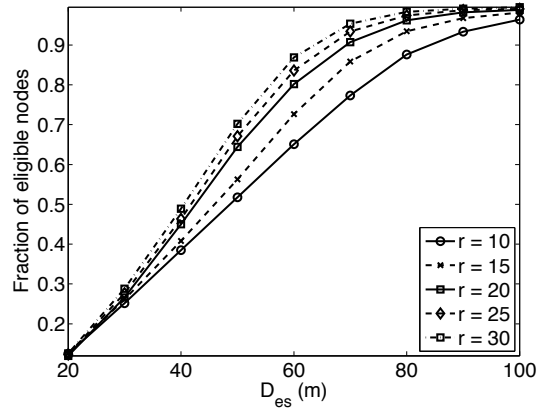

(c)

Fig. 3: Fraction of eligible nodes in the network vs. $D_{\text {es }}$ for (a) different $\mathrm{R}(\mathrm{m})$ when $\mathrm{r}=20 \mathrm{~m}$ and $\mathrm{N}=200$, (b) different $\mathrm{N}$ when $\mathrm{r}=20 \mathrm{~m}$ and $\mathrm{R}=30 \mathrm{~m}$, (c) different $\mathrm{r}(\mathrm{m})$ when $\mathrm{R}=20 \mathrm{~m}$ and $\mathrm{N}=200$

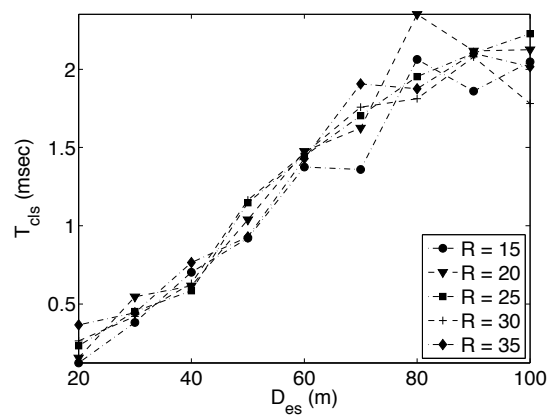

(a)

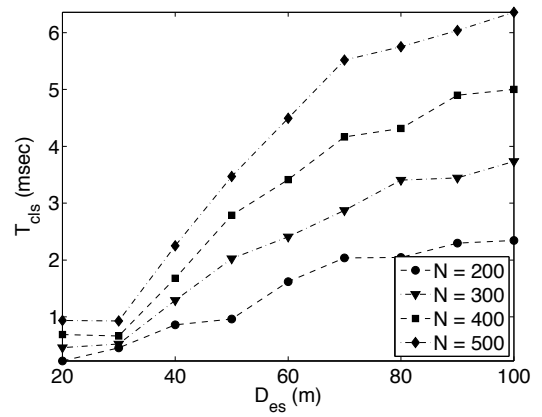

(b)

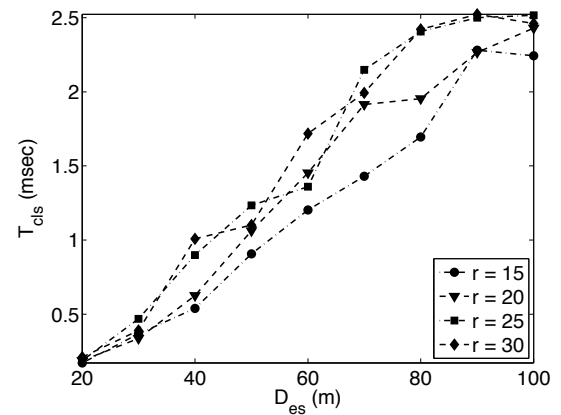

(c)

Fig. 4: $T_{c l s}(\mathrm{msec})$ vs. $D_{e s}(\mathrm{~m})$ for (a) different $\mathrm{R}(\mathrm{m})$ when $\mathrm{r}=20 \mathrm{~m}$ and $\mathrm{N}=200$, (b) different $\mathrm{N}$ when $\mathrm{R}=30 \mathrm{~m}$ and $\mathrm{r}=$ $20 \mathrm{~m}$, (c) different $\mathrm{r}(\mathrm{m})$ when $\mathrm{R}=20 \mathrm{~m}$ and $\mathrm{N}=200$

\section{A. Ratio of eligible nodes in the network}

The most important advantage of our protocol is that clustering does not take place in the entire network but occurs in the event region in addition to the corridor between event and sink. By changing the network parameters, fraction of the eligible nodes in the network is examined. Throughout the simulations, sink is placed at $\mathrm{X}=0$ and $\mathrm{Y}=50$ in the network field. In the simulations, the results are averaged over 50 different topologies. In the first simulation setup, by increasing the distance between event and sink, we investigate the ratio of the eligible nodes with different event radius. Event is generated on the $\mathrm{Y}=50$ line, $200 \mathrm{CR}$ nodes are distributed uniformly and transmission range of nodes is set to 20. Fig. 3a shows that only under $30 \%$ of the nodes in the network join the clustering when the event to sink distance is 20 for an event radius 30 . If event radius is 10 , the fraction is below $10 \%$. The fraction goes over $90 \%$ if event radius is 30 and the distance between event and sink is above 60 . For this case, due to large event radius substantial number of nodes whose distances to sink are above 60 become eligible, and those nodes and their neighbors increase the fraction of eligible nodes. On the other hand, for events with radius of 10 , even if distance between the event and the sink is 60, fraction of eligible nodes is $55 \%$. As seen from Fig. 3a, considerable number of nodes do not join clustering for small event to sink distances, hence these nodes do not consume energy due to clustering.

In the second simulation setup, in a 100 x 100 network field, different number of CRs are deployed uniformly while event radius is 30 and transmission range of CR is 20. For this setup, the fraction of eligible nodes does not change significantly since CR transmission range which is 20 does not change the region between event and sink for different number of nodes. Fig. $3 b$ shows this effect such that fractions of eligible nodes are almost the same for different number of nodes and for CR transmission range 20 .

In the third simulation setup, variation of the transmission range of nodes on the ratio of the eligible nodes is investigated. $200 \mathrm{CR}$ nodes are distributed uniformly and event radius is fixed to be 20. As shown in Fig. 3c, rise in the transmission range of $\mathrm{CR}$ increases the number of eligible nodes. An eligible node makes more of its neighbor nodes eligible since its node degree increases due to rise in transmission range. More eligible neighbor nodes mean higher of fraction of eligible nodes in the network.

In the simulations, we observe that the increase in $\mathrm{CR}$ transmission range and event radius causes raise in the fraction of eligible nodes in the network. On the other hand, increase in network density does not change the fraction since trans- 


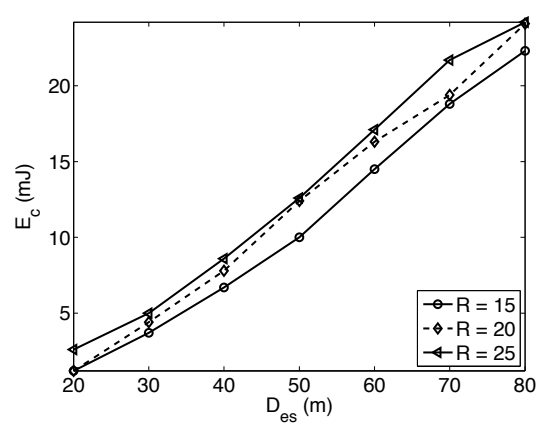

(a)

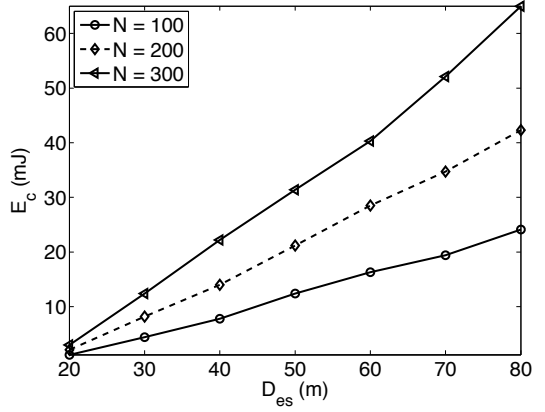

(b)

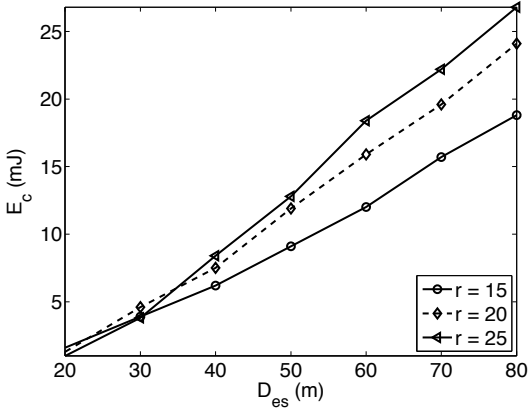

(c)

Fig. 5: $E_{c}(\mathrm{~mJ})$ vs. $D_{e s}(\mathrm{~m})$ for (a) different $\mathrm{R}(\mathrm{m})$ when $\mathrm{r}=20 \mathrm{~m}$ and $\mathrm{N}=100$, (b) different $\mathrm{N}$ when $\mathrm{R}=20 \mathrm{~m}$ and $\mathrm{r}=20$ $\mathrm{m}$, (c) different $\mathrm{r}(\mathrm{m})$ when $\mathrm{R}=20 \mathrm{~m}$ and $\mathrm{N}=200$

mission range of $\mathrm{CR}$ is enough to determine the same region between event and sink for different network densities. Since the nodes are uniformly distributed and the area of the eligible nodes does not change, the fractions for different network densities are the same.

\section{B. Delay, Energy and Connectivity}

In this section, we investigate our protocol performance in terms of delay to form clusters, energy consumed due to control signaling between eligible nodes and the connectivity between cluster-head and its two-hop neighbors. For this simulation setup, control channel's bandwidth is assumed to be $512 \mathrm{kbps}$ [27] and the length of the control packet is 200 bits. The environment is collision free in order to realize the effect of control signaling only. There are 10 orthogonal nonoverlapping channels. 200 nodes and 20 primary users are distributed uniformly over an area $100 \times 100$. Transmission range of a $\mathrm{PU}$ is $30 \mathrm{~m}$.

In Fig. 4, the cumulative delay for different scenarios due to cluster formation is investigated. In this process, determining eligible nodes and forming clusters are the causes of the cumulative delay, i.e., clustering time $\left(T_{c l s}\right)$. If an eligible node $i$ knows the eligibility condition of its one-hop neighbors by EFC_REP messages, it determines whether it is a cluster-head according to its weight $P_{i}$. If it is cluster-head, it computes its clustering set according to Algorithm 2. It asks the nodes in clustering set to join its cluster. The nodes accepting to be a member of that cluster notify the cluster-head. As the event radius increases, time required to form clusters do not differ considerably for different event to sink distance as shown in Fig. 4a. Event radius is not important for the delay since the nodes in the event region are immediately start to form cluster due to becoming eligible after detecting the event. In this case as the distance between event and sink increases, the time required to form clusters rises since number of hops between event and sink increases. In Fig. $4 b$, the rise in node density increases the number of eligible nodes, and this raises the clustering time since more nodes are required to exchange control signals to form clusters. Unlike fraction of eligible nodes, clustering time rises for increasing node density. In Fig. 4c, for different CR transmission range, delay changes. The increase in number of neighbors with increasing transmission range results in more nodes to be clustered. Therefore, clustering time for $\mathrm{r}=15$ is smallest among the others and the clustering time for $r=30$ is generally higher than the others. Fig. 4 shows that clustering time increases significantly with respect to number of nodes $(\mathrm{N})$ and distance between event and sink $\left(D_{e s}\right)$.

In our scheme, we try to establish a coordination among the nodes between event and sink for each event occurrences. This requires energy consumption for this setup. In our simulations, radio model in [26] is adopted such that $\epsilon_{f s}=10 \mathrm{pJ} / \mathrm{bit} / \mathrm{m}^{2}$ and $E_{\text {elec }}=50 \mathrm{~nJ} /$ bit. We investigate total energy consumption for coordination by changing event to sink distance and other network parameters. In energy consumption scenario, 200 nodes are deployed randomly in network field. Fig. 5a shows slight increase in $E_{c}$. It is the natural result of the Fig. 3a since more nodes are performing clustering operations due to increase in event radius. As shown in Fig 5b, control signal transmissions increase since more nodes join clustering as network density rises. If we raise the $\mathrm{CR}$ transmission range, additional nodes become eligible. Hence, there is slight increase in total consumed energy especially for $D_{e s}$ greater than $30 \mathrm{~m}$ as shown in Fig. 5c.

We also perform simulations to study the energy consumption of the nodes to form clusters during an event process that is generated at $\mathrm{X}=50 \mathrm{~m}$ and $\mathrm{Y}=50 \mathrm{~m} .200 \mathrm{CR}$ nodes and $20 \mathrm{PU}$ nodes are distributed randomly and there are 10 licensed channels. Transmission ranges of PU and CR are 30 and 15 , respectively. According to this setup, average energy consumed per event by a node in ESAC is less than SOC as shown in Fig. 6 since SOC forms clusters in the entire region and it is more prone to re-clustering due to dynamic radio environment. In ESAC, clusters are formed in the corridor between event and sink and not maintained after the event. ESAC uses network resources when communication exists in the region between event and sink, hence, our protocol is more appropriate in terms of energy consumption for sensor networks which are event based systems.

Connectivity of clusters is an important metric for valid 


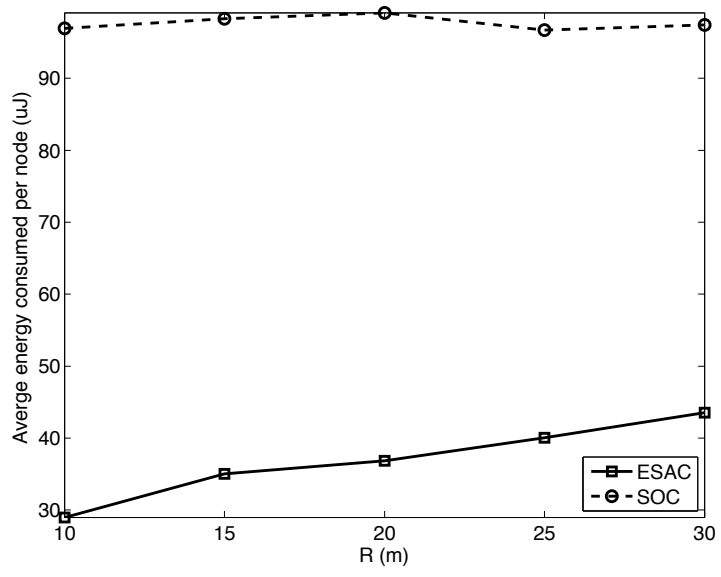

Fig. 6: Average energy consumed per node $(\mu \mathrm{J})$ vs. R (m) (N $=200$ and $\mathrm{r}=15 \mathrm{~m}$ )

cluster formations. We define the average cluster connectivity as the average number of two-hop nodes that a clusterhead can communicate with by its members through cluster channels normalized by total eligible nodes in the network. Our algorithm aims to maximize the number of two-hop neighbors which are the members of neighbor clusters having common channels with the cluster-head. In this simulation setup, event is generated at $\mathrm{X}=100 \mathrm{~m}$ and $\mathrm{Y}=50 \mathrm{~m}, \mathrm{CR}$ transmission range is varied during simulation. Fig. 7 shows that our algorithm performs better than the SOC algorithm if it is applied in ESAC configuration. In other words, not considering two-hop neighbors in ESAC results in degradation in connectivity. The condition of maximizing accessible two hop members for connectivity make ESAC performance better than SOC algorithm used in ESAC configuration.

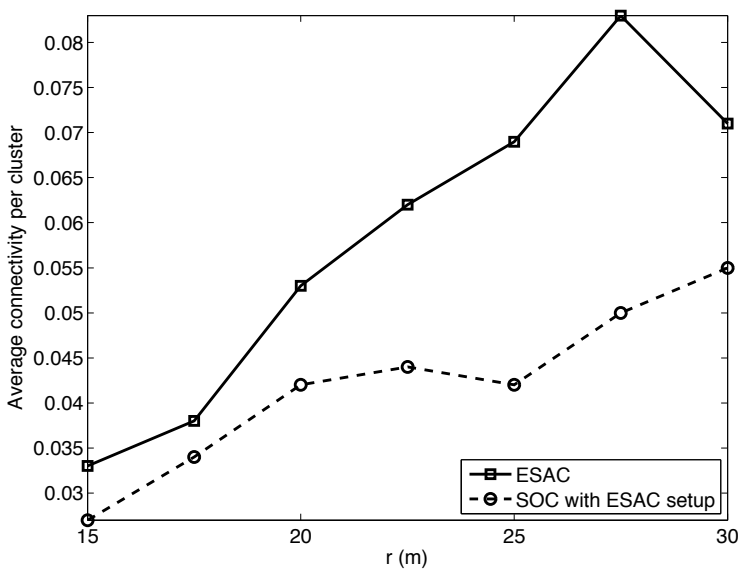

Fig. 7: Average connectivity per cluster vs. $\mathrm{r}(\mathrm{m})(\mathrm{N}=200$ and $\mathrm{R}=20 \mathrm{~m}$ )

\section{CONCLUSION}

In this paper, we present a clustering protocol for cognitive radio sensor networks (CRSN) in order to minimize the energy consumption for delay tolerant systems. By clustering, we aim to manage spectrum holes and establish energy-efficient communication. The most important features of our protocol are that clustering is event-driven and in the corridor between event and sink. Furthermore, in our clustering protocol, we try to establish a compromise between cluster size, common channels and two-hop neighbors that can be reachable by cluster-head through its members. Performance evaluation shows that ESAC is energy-efficient with a delay caused by spontaneous cluster formation.

\section{ACKNOWLEDGMENT}

This work was supported in part by the Turkish Scientific and Technical Research Council under grant \#110E249 and by the Turkish National Academy of Sciences Distinguished Young Scientist Award Program (TUBA-GEBIP).

\section{REFERENCES}

[1] S. Haykin, "Cognitive Radio: Brain-empowered Wireless Communications," IEEE Journal on Selected Areas in Communications (JSAC), Vol. 23, No. 2, pp. 201-220, Feb. 2005.

[2] I. F. Akyildiz, W. Y. Lee, M.C. Vuran, and S. Mohanty, "NeXt Generation/Dynamic Spectrum Access/Cognitive Radio Wireless Networks: A Survey," Comput. Networks (Elsevier), vol. 50, no. 13, pp. 2127-2159, Sep. 2006.

[3] O. B. Akan, O. B. Karli, and O. Ergul, "Cognitive Radio Sensor Networks," IEEE Network, vol. 23, no. 4, pp. 34-40, Jul.-Aug. 2009.

[4] J. A. Han, W. S. Jeon, and D. G. Jeong, "Energy-efficient Channel Management Scheme for Cognitive Radio Sensor Networks," IEEE Transactions on Vehicular Technology, vol. 60, no. 4, pp. 1905-1910, May 2011.

[5] M. C. Oto, and O. B. Akan, "Energy-Efficient Packet Size Optimization for Cognitive Radio Sensor Networks," IEEE Transactions on Wireless Communications, vol. 11, no. 4, pp. 1544-1553, Apr. 2012.

[6] A. O. Bicen, and O. B. Akan, "Reliability and Congestion Control in Cognitive Radio Sensor Networks," Ad Hoc Netw. J., vol. 9, no. 7, pp. 1154-1164, Sep. 2011.

[7] J. Y. Yu, and P. H. J. Chong, "A Survey of Clustering schemes for mobile ad hoc networks," IEEE Communications Surveys \& Tutorials, vol. 7, no. 1, pp. 32-48, First Qtr. 2005.

[8] C. R. Lin, and M. Gerla, "Adaptive Clustering for Mobile Wireless Networks," IEEE Journal on Selected Areas in Communications, vol. 15, pp. 1265-1275, Sept. 1997.

[9] S. Basagni, "Distributed Clustering for Ad Hoc Networks," in Proc. ISPAN 99, pp. 310-315, Fremantle, Australia, Jun. 1999.

[10] V. Kawadia and P. Kumar,"Power Control and Clustering in Ad $\mathrm{Hoc}$ Networks," in Proc. 22nd IEEE Conf. Comput. Commun., pp. 459-469, San Francisco, CA, USA, 2003.

[11] M. Chatterjee, S. K. Das, and D. Turgut, "WCA: A Weighted Clustering Algorithm for Mobile Ad hoc Networks," Journal of Cluster Computing, Special issue on Mobile Ad hoc Networking, No. 5, pp. 193-204, 2002.

[12] S. Bandyopadhyay, and E. J. Coyle, "An Energy Efficient Hierarchical Clustering Algorithm for Wireless Sensor Networks," in Proc. INFOCOM 2003, vol.3, pp. 1713-1723, San Francisco, CA, USA, 30 March-3 April 2003.

[13] M. A. Youssef, A. Youssef, and M. F. Younis, "Overlapping Multihop Clustering for Wireless Sensor Networks," IEEE Transactions on Parallel Distributed Systems, vol. 20, no. 12, pp. 1844-1856, Dec. 2009.

[14] D. Baker, and A. Ephremides, "The Architectural Organization of a Mobile Radio Network via a Distributed Algorithm," IEEE Transactions on Communications, vol. 29, no. 11, pp. 1694-1701, Nov 1981.

[15] O. Younis, and S. Fahmy, "HEED: A Hybrid, Energy-Efficient, Distributed Clustering Approach for Ad Hoc Sensor Networks," IEEE Transactions on Mobile Computing, vol. 3, no. 4, pp. 366-379, Oct.-Dec. 2004.

[16] P. Ding, J. Holliday, and A. Celik, "Distributed Energy Efficient Hierarchical Clustering for Wireless Sensor Networks," in Proceedings of the IEEE International Conference on Distributed Computing in Sensor Systems(DCOSS'05), Marina Del Rey, CA, USA, Jun. 2005. 
[17] A. Bereketli, and O. B. Akan, "Event-to-Sink Directed Clustering in Wireless Sensor Networks," in Proc. IEEE WCNC 2009, Budapest, Hungary, Apr. 2009.

[18] J. Zhao, H. Zheng, and G. Yang, "Distributed Coordination In Dynamic Spectrum Allocation Networks," in Proc. IEEE DySPAN 2005, Baltimore, MD, USA, Nov. 2005

[19] T. Chen, H. Zhang, G. M. Maggio, and I. Chlamtac, "Cogmesh: A Cluster-based Cognitive Radio Network," in Proc. IEEE DySPAN 2007, pp. 168-178, Dublin, Ireland, Apr. 2007.

[20] L. Lazos, S. Liu, and M. Krunz, "Spectrum Opportunity-based Control Channel Assignment in Cognitive Radio Networks," in Proc. IEEE SECON 2009, pp. 135-143, Rome, Italy, Jun. 2009.

[21] M. Bradonjic, and L. Lazos, "Graph-based Criteria for Spectrum-aware Clustering in Cognitive Radio Networks," Ad Hoc Networks, Volume 10, Issue 1, Pages 75-94, Jan. 2012.

[22] K. E. Baddour, O. Ureten and T. J. Willink, "Efficient Clustering of Cognitive Radio Networks Using Affinity Propagation," in Proc. 18th Int. Conf. Comp. Commun. and Networks, San Francisco, CA, USA, Aug. 2009.
[23] D. Li, and J. Gross, "Robust Clustering of Ad-Hoc Cognitive Radio Networks under Opportunistic Spectrum Access," in Communications (ICC), 2011 IEEE International Conference on, pp.1-6, Kyoto, Japan, Jun. 2011.

[24] H. Zhang, Z. Zhang, H. Dai, R. Yin, and X. Chen, "Distributed Spectrum-Aware Clustering in Cognitive Radio Sensor Networks," in Proc. IEEE Globecom 2011, , Houston, TX, USA, Dec. 2011.

[25] J. Jia, Q. Zhang, and X. Shen, "HC-MAC: A Hardware-Constrained Cognitive MAC for Efficient Spectrum Management," IEEE Journal on Selected Areas in Communications, vol. 26, no. 1, pp. 106-117, Jan. 2008.

[26] W. R. Heinzelman, A. Chandrakasan, and H. Balakrishnan, "EnergyEfficient Communication Protocol for Wireless Microsensor Networks," in Proceedings of the 33rd Hawaii International Conference on System Sciences, pp. 1-10, 2000.

[27] G. A. Shah, and O. B. Akan, "CSMA-Based Bandwidth Estimation for Cognitive Radio Sensor Networks," in Proc. 5th IFIP/IEEE NTMS 2012, pp.1-5, Istanbul, Turkey, May 2012. 